\title{
Identification and Characterization of the Corazonin Receptor and Possible Physiological Roles of the Corazonin-Signaling Pathway in Rhodnius prolixus
}

\author{
Zina Hamoudi *, Angela B. Lange and lan Orchard \\ Department of Biology, University of Toronto Mississauga, Mississauga, ON, Canada
}

\section{OPEN ACCESS}

Edited by:

Liliane Schoofs,

Catholic University of Leuven, Belgium

Reviewed by:

Young-Joon Kim,

Gwangju Institute of Science and

Technology, South Korea

Gert Jansen,

Erasmus University Rotterdam,

Netherlands

${ }^{*}$ Correspondence:

Zina Hamoud

zina.hamoudi@mail.utoronto.ca

Specialty section

This article was submitted to

Neuroendocrine Science,

a section of the journal

Frontiers in Neuroscience

Received: 07 May 2016

Accepted: 18 July 2016

Published: 03 August 2016

Citation:

Hamoudi Z, Lange AB and Orchard

(2016) Identification and

Characterization of the Corazonin

Receptor and Possible Physiological

Roles of the Corazonin-Signaling

Pathway in Rhodnius prolixus.

Front. Neurosci. 10:357.

doi: 10.3389/fnins.2016.00357
Neuropeptides control many physiological and endocrinological processes in animals, acting as neuroactive chemicals within the central and peripheral nervous systems. Corazonin (CRZ) is one such neuropeptide that has a variety of physiological roles associated with control of heartbeat, ecdysis behavior initiation, and cuticle coloration. These physiological effects are mediated by the CRZ receptor (CRZR). In order to understand the role of the CRZ-signaling pathway in Rhodnius prolixus, the cDNA sequence encoding the Rhopr-CRZR was isolated and cloned revealing two splice variants (Rhopr-CRZR- $\alpha$ and $\beta$ ). Sequence analysis revealed characteristics of rhodopsin-like GPCRs. Rhopr-CRZR- $\alpha$ and $\beta$ were dose-dependently activated by Rhopr-CRZ with $\mathrm{EC}_{50}$ values of 2.7 and $1 \mathrm{nM}$, respectively, when tested in a functional receptor assay using $\mathrm{CHOKI}$-aeq cells. Neither receptors were activated by the evolutionarily-related peptides, Rhopr-AKH, or Rhopr-ACP. For 5th instars, qPCR revealed expression of Rhopr-CRZR transcript in the CNS, the dorsal vessel, abdominal dorsal epidermis, and prothoracic glands with associated fat body. Interestingly, transcript expression was also found in the female and male reproductive tissues. Rhopr-CRZR transcript was reduced after injection of dsCRZR into adult $R$. prolixus. In these insects, the basal heartbeat rate was reduced in vivo, and the increase in heartbeat frequency normally produced by CRZ on dorsal vessel in vitro was much reduced. No effect of dsCRZR injection was seen on ecdysis or coloration of the cuticle.

Keywords: insect, peptide, corazonin, GPCR, dsRNA, heartbeat, ecdysis, cuticle color

\section{INTRODUCTION}

Fundamental questions in physiology revolve around understanding the means by which the nervous system communicates information (messages) throughout the organism. To do this, neurons use a variety of chemical messengers that act as neurotransmitters, neuromodulators, and neurohormones to allow for flexibility in the privacy, speed, and length of the message (Orchard et al., 2001). These chemical messengers often transduce their message via receptors that are located in the membrane on the target cell. The use of model organisms to study these signaling pathways is advantageous particularly since the genomes of a variety of organisms have been sequenced, 
allowing for the use of molecular tools to examine these pathways and their messengers; the most diverse messengers being via neuropeptides. These neuropeptides are often associated with distinct behaviors, although piecing together the overall integration of such behaviors is a challenge in neurobiology (Orchard et al., 2001).

The corazonin (CRZ)-signaling pathway is one such neuropeptide system that appears to have diverse functions throughout a variety of insect species despite CRZ having a conserved sequence (Veenstra, 2009). [ $\left.\mathrm{Arg}^{7}\right]-\mathrm{CRZ}$, with the sequence PQTFQYSRGWTNamide, is the most abundant CRZ sequence present in insects (Veenstra, 1989, 1991; Hua et al., 2000). It has been proposed that this signaling pathway arose during protostome evolution alongside two other insect signaling pathways, the adipokinetic hormone $(\mathrm{AKH})$, and the AKH/CRZrelated peptide (ACP) pathways (Hauser and Grimmelikhuijzen, 2014; Roch et al., 2014). Utilizing genomic data and using an in silico approach, Hauser and Grimmelikhuijzen (2014) proposed that an ancestral gonadotropin releasing hormone ( $\mathrm{GnRH})$-like peptide and its receptor duplicated and diverged before the emergence of Mollusca and Annelida leading to an AKH-like peptide and receptor, as well as a CRZ-like peptide and receptor. Before the emergence of the Arthropoda, the AKH-signaling system duplicated leading to AKH and ACP systems along with the CRZ-signaling system (Roch et al., 2014).

CRZ increases the heartbeat rate in the cockroach Periplaneta americana (Veenstra, 1989), and recently was shown to do the same in $R$. prolixus (Patel et al., 2014). A study on Anopheles gambiae, however, showed that when $\mathrm{CRZ}$ and its receptor were knocked down by RNA interference (RNAi), there was no significant effect on heartbeat rate (Hillyer et al., 2012). In Manduca sexta, CRZ was found to initiate ecdysis behavior (Kim et al., 2004); however, this effect has not been reported in other insects. In locusts CRZ was found to induce dark body coloration (Tanaka, 1993; Tanaka and Pener, 1994; Tanaka and Yagi, 1997; Tawfik et al., 1999). When light colored solitarious nymphs were injected with [His $\left.{ }^{7}\right]$-CRZ, Schistocerca gregaria (Tawfik et al., 1999), and Locusta migratoria (Tanaka, 2000) both developed black patterns; however, again CRZ did not have such an effect when tested in other insects such as Galleria mellonella, Gryllus bimaculatus, or Bombyx. mori (Hua et al., 2000; Hansen et al., 2010).

The physiological functions of CRZ are mediated by a signaltransducing membrane receptor, a $G$ protein-coupled receptor (GPCR). All GPCRs possess a similar topographical structure that has been well-conserved through evolution (Caers et al., 2012). They all have seven transmembrane spanning helices each consisting of 20-30 amino acids, connected by three intracellular loops, and three extracellular loops. Moreover, they have an extracellular N-terminus, which holds several glycosylation sites, and an intracellular C-terminus with potential phosphorylation sites. The ligand binds to the extracellular part of the receptor, activating it and eliciting an intracellular response. Based on phylogenetic analysis, GPCRs can be classified into at least five subfamilies: rhodopsin, secretin, glutamate, adhesion and frizzled-tastes-2 (Fredriksson et al., 2003). The CRZ receptor (CRZR) belongs to the family of rhodopsin-like receptors, the largest subfamily of GPCRs. They are characterized by having a DRY motif at the border of the cytoplasmic end of the third transmembrane domain and a NSxxNPxxY domain in transmembrane seven (Oldham and Hamm, 2008). These motifs are believed to be important for $G$ protein activation and/or protein stabilization (Ballesteros et al., 2001). To date, the CRZR has been cloned and characterized in the following insects: Drosophila melanogaster (Cazzamali et al., 2002; Park et al., 2002), M. sexta (Kim et al., 2004), A. gambiae (Belmont et al., 2006), and B. mori (Yang et al., 2013). The CRZR has also been cloned in Musca domestica (Sha et al., 2012).

Rhodnius prolixus, the vector of Chagas disease, is an ideal model for studying the control of physiological processes since many of these processes are driven by gorging on a blood meal once in each instar. This blood gorging then stimulates shortterm physiological changes associated with digestion, and the elimination of excess salt and water, and long-term changes associated with growth and development. In the adult, blood gorging stimulates reproductive activity. Thus, these events can be initiated by a blood meal and accurately timed. In addition, the genome of $R$. prolixus was recently sequenced (Mesquita et al., 2015) enabling further analysis of signaling pathways associated with physiological and endocrinological processes. This paper examines the CRZ-signaling pathway in $R$. prolixus, cloning and de-orphaning the receptor and then manipulating its expression to gain insight into the physiological relevance of CRZ in R. prolixus.

\section{MATERIALS AND METHODS}

\section{Animals}

Fifth instar and adult $R$. prolixus were maintained in incubators at $60 \%$ humidity and $25^{\circ} \mathrm{C}$. They were routinely fed once at each instar stage on defibrinated rabbits' blood (Hemostat Laboratories, Dixon, CA, USA; supplied by Cedarlane Laboratories Inc., Burlington, ON, Canada).

\section{Isolating and Cloning the cDNA Sequence Encoding Rhopr-CRZR}

Supercontig sequences representing the $R$. prolixus genome were imported to Geneious 4.7.6 to perform a local tBLASTn search with D. melanogaster CRZR (JC7896) amino acid sequence to mine for the CRZR in R. prolixus. Gene-specific primers, CRZRFOR1 and CRZR-REV1 (Supplementary Table 1) were designed and used to amplify the partial cDNA sequence encoding RhoprCRZR using $R$. prolixus CNS cDNA as a template. The PCR reaction was performed with an S1000 thermal cycler (BioRad Laboratories, Mississauga, ON, Canada) using the following temperature-cycling profiles: initial denaturation at $94^{\circ} \mathrm{C}$ for 3 min, followed by 39 cycles of $94^{\circ} \mathrm{C}$ for $30 \mathrm{~s}, 60^{\circ} \mathrm{C}$ for $30 \mathrm{~s}$, and $72^{\circ} \mathrm{C}$ for $1 \mathrm{~min}$, followed by a final extension at $72^{\circ} \mathrm{C}$ for $10 \mathrm{~min}$. The PCR product was then column purified using the Axygen ${ }^{\mathrm{TM}}$ Axyprep $^{\mathrm{TM}}$ PCR Clean-up Kit (Fisher Scientific Ltd., Ottawa, ON, Canada). The purified product was then cloned using pGEMT Easy Vector (Promega, Madison, WI, USA). Positive clones containing the desired inserts were purified from an overnight culture using the PureLink ${ }^{\circledR}$ Quick Plasmid Miniprep Kit (Life 
Technologies, Carlsbad, CA, USA) and sent for sequencing at the Centre of Applied Genomics at the Hospital for Sick Children (MaRS Centre, Toronto, Ontario, Canada).

The complete $3^{\prime}$ end of Rhopr-CRZR cDNA was obtained using a modified $3^{\prime}$ rapid amplification of cDNA ends (RACE) PCR approach using fifth-instar $R$. prolixus CNS cDNA library (Paluzzi et al., 2008). Gene specific primers were designed using the open reading frame (ORF) and used in combination with plasmid-specific primers (Supplementary Table 1). A series of nested PCRs was performed in succession using three gene-specific forward primers (CRZR-RACE-FOR1, CRZRRACE-FOR2, and CRZR-RACE-FOR3) and two plasmid-specific reverse primers (pDNR-LIB 3 -88 REV and pDNR-LIB 3 $25 \mathrm{REV}$ ) (Supplementary Table 1). The PCR product of each reaction was purified and used for the subsequent PCR reaction. The final RACE product was cloned and sequenced as described earlier.

Using the $R$. prolixus genome (Mesquita et al., 2015), a gene-specific primer was designed to obtain the full $5^{\prime}$ end of the Rhopr-CRZR cDNA sequence. To confirm the sequences obtained from the PCR reactions, CRZR-FOR0, and CRZR-REV4 (Supplementary Table 1) were used to amplify the largest RhoprCRZR cDNA fragment. The resulting PCR product was also cloned and sequenced as described earlier. Multiple independent clones were sequenced to ensure base accuracy.

\section{Rhopr-CRZR Sequence Analysis}

The seven transmembrane domains of the RhoprCRZR were predicted using TMHMM Server v. 2.0 (http://www.cbs.dtu.dk/services/TMHMM/). The exon-intron boundaries were predicted using BLAST and confirmed with a splice site prediction software (http://www.fruitfly.org/seq_tools/splice.html). The Nlinked glycosylation sites and intracellular phosphorylation sites were predicted using NetGlyc 1.0 Server (http://www.cbs.dtu.dk/services/NetNGlyc/) and NetPhos 2.0 Server (http://www.cbs.dtu.dk/services/NetPhos/), respectively. The RhopCRZR amino acid sequence was aligned with the sequences from the following cloned CRZRs: D. melanogaster (JC7896), M. sexta (AAR14318.1), A. gambiae (AY301275), $M$. domestica (AEI91710.1), and B. mori (NP-001127719.1) using MAFFT (http://mafft.cbrc.jp/alignment/server/index.html).

\section{Preparation of Expression Vector and Receptor Functional Assay}

The ORF of the two Rhopr-CRZR variants were amplified and a Kozak translation initiation sequence was introduced at the $5^{\prime}$ end using primers listed in Supplementary Table 2. The resulting product was cloned into $\mathrm{PGEM}$-T Easy vector and subcloned into pIRES2-ZsGreen1 (Clontech, Mountain View, CA, USA).

Chinese hamster ovary (CHO) cells stably expressing aequorin (CHOK1-aeq) (Paluzzi et al., 2010) were cultured in complete media (94\% Dulbecco's Modified Eagle Medium Nutrient Mixture F12-Ham (DMEM/F12) (Life Technologies Corporation, Carlsbad, CA, USA), 5\% heat-inactivated fetal bovine serum (FBS), and 1\% penicillin/streptomycin) containing $200 \mu \mathrm{g} / \mathrm{mL}$ Geneticin. The cells were incubated at $37^{\circ} \mathrm{C}$ in $5 \%$
$\mathrm{CO}_{2}$, and then transiently transfected with the expression vector for the receptor transcript variants using X-tremeGENE 9 DNA transfection reagent (Roche Applied Science, Indianapolis, IN, USA) at a ratio of 2:1 (transfection reagent to expression vector) using the manufacturer recommended protocol. Cells were also transiently transfected with an empty expression vector as a negative control.

The bioluminescence assay was performed $72 \mathrm{~h}$ posttransfection. The cells were first harvested with PBS/EDTA solution and suspended in bovine serum albumin BSA media (DMEM/F12 containing $1 \%$ BSA and $1 \%$ penicillin/streptomycin). The cells were incubated in the dark for $4-5 \mathrm{~h}$ at room temperature with $5 \mu \mathrm{M}$ (final concentration) coelenterazine h (Promega, Madison, WI, USA) and then diluted 10 -fold using BSA media. Various peptide concentrations were prepared with BSA media and loaded in triplicates on an opaque 96-well microplate. Cells were loaded into each well using an automated injector unit and luminescence was recorded over $15 \mathrm{~s}$ using a Wallac Victor2 plate reader (Perkin Elmer, San Diego, CA, USA). The peptides used were Rhopr-CRZ (pQTFQYSRGWTNamide), Rhopr-AKH (pQLTFSTDWamide), and Rhopr-ACP (pQVTFSRDWNAamide). Wells containing only the BSA media served as a blank control while wells containing 25, 50, and $100 \mu \mathrm{M}$ ATP served as positive controls. Dose-response curves were obtained by averaging three replica plates and the $\mathrm{EC}_{50}$ values were determined using Prism5 software.

\section{Spatial Expression Pattern of Rhopr-CRZR Using Quantitative PCR (qPCR)}

Rhopr-CRZR transcript expression was examined in various tissues from 5 th instar $R$. prolixus. Tissues were dissected from 4 to 6 weeks post-fed (as previous instar) insects in nucleasefree phosphate-buffered saline (PBS) (Sigma-Aldrich, Oakville, ON, Canada). To determine the expression of the Rhopr-CRZR transcript around ecdysis, the CNS was dissected from 4th instar $R$. prolixus 3 days before ecdysis (DBE), $2 \mathrm{DBE}, 1 \mathrm{DBE}, 2 \mathrm{~h}$ postecdysis (2 HPE) into 5th instar, $4 \mathrm{HPE}, 1$ day post-ecdysis (DPE), 3 DPE, and 6 DPE.

Total RNA was extracted from each tissue using PureLink ${ }^{\circledR}$ RNA Mini Kit (Life Technologies Corporation, Carlsbad, CA, USA) which was then used for cDNA synthesis with iScript ${ }^{\mathrm{TM}}$ Reverse Transcription Supermix for RT-qPCR (BioRad Laboratories Ltd., Mississauga, ON, Canada). The cDNA was diluted 10 -fold and used as a template for the qPCR reaction. The Rhopr-CRZR gene and the reference genes (alpha-tubulin, betaactin, and ribosomal protein 49) were amplified using primers designed over exon/exon boundaries (Supplementary Table 3; Paluzzi et al., 2010; Paluzzi and O’Donnell, 2012). The qPCR reactions were carried out on the CFX384 Touch $^{\text {TM }}$ Real-Time PCR Detection System (Bio-Rad, Mississauga, ON, Canada) using SsoFAST ${ }^{\mathrm{TM}}$ EvaGreen ${ }^{\circledR}$ Supermix (Bio-Rad Laboratories Ltd., Mississauga, ON, Canada). The amplification conditions were as follows: initial denaturation at $95^{\circ} \mathrm{C}$ for $30 \mathrm{~s}, 40$ cycles of denaturation at $95^{\circ} \mathrm{C}$ for $5 \mathrm{~s}$, annealing at $60^{\circ} \mathrm{C}$ for $5 \mathrm{~s}$, and extension at $72^{\circ} \mathrm{C}$ for $5 \mathrm{~s}$. Three biological replicates with three 
technical replicates were performed including a no-template control. The melting curve analysis was performed and the qPCR products were run on a gel and cloned to confirm that the specific transcript was amplified. The relative expression levels were determined using the $\Delta \Delta \mathrm{Ct}$ method and the fold differences were normalized to the three reference genes using the geometric averaging of the transcript levels.

\section{Fluorescent In situ Hybridization}

The distribution of cells expressing the Rhopr-CRZR mRNA within the brain was determined using fluorescent in situ hybridization (FISH). To synthesize the sense and antisense probes, Rhopr-CRZR was amplified via PCR using CNS cDNA as a template. T7 promoter sequence at the $5^{\prime}$ end of the sense strand and the $3^{\prime}$ end of the antisense strand was added to the PCR products using the primers listed in Supplementary Table 4. The PCR amplification conditions were as follows: initial denaturation at $94^{\circ} \mathrm{C}$ for $3 \mathrm{~min}$, followed by seven cycles of $94^{\circ} \mathrm{C}$ for $30 \mathrm{~s}, 58^{\circ} \mathrm{C}$ for $30 \mathrm{~s}$ and $72^{\circ} \mathrm{C}$ for $90 \mathrm{~s}, 30$ cycles of $94^{\circ} \mathrm{C}$ for $30 \mathrm{~s}$, $62^{\circ} \mathrm{C}$ for $30 \mathrm{~s}$ and $72^{\circ} \mathrm{C}$ for $90 \mathrm{~s}$, and a final extension at $72^{\circ} \mathrm{C}$ for $10 \mathrm{~min}$. The PCR products were purified and were labeled using the DIG RNA Labeling Kit (Roche Applied Science, Mannheim, Germany) following the manufacturer's protocol and stored at $-20^{\circ} \mathrm{C}$ until use.

The brains were dissected in nuclease-free phosphate-buffered saline (PBS) and fixed in 4\% paraformaldehyde prepared in PBST (PBS containing $0.1 \%$ Tween) for $1 \mathrm{~h}$ at room temperature. FISH was performed as previously described (Defferrari et al., 2016) using 150 ng of the anti-sense, or sense for controls, DIGlabeled Rhopr-CRZR RNA probes. No staining was observed in the controls. The cell-specific spatial expression of the transcript was viewed under a Zeiss Laser scanning confocal microscope LSM510 and LSM image browser software (Carl Zeiss, Jena, Germany).

\section{Double Stranded RNA Synthesis and Delivery}

The RNA probes were synthesized in a similar manner to the FISH probes. Rhopr-CRZR was used as a template for the experimental dsRNA whereas pGEM-T East vector (Promega, Madison, WI, USA) was used as a template for the control, ampicillin resistance gene (ARG). The dsRNA was synthesized and purified using the T7 Ribomax Express RNAi System (Promega, Madison, WI, USA), following the manufacturersupplied protocol.

Adult $R$. prolixus were anesthetized briefly with $\mathrm{CO}_{2}$ and injected with $2 \mu \mathrm{L}$ of $2 \mu \mathrm{g} / \mu \mathrm{L}$ of dsCRZR or dsARG into the thorax using a $5 \mu \mathrm{L}$ Hamilton syringe. Insects were left for $30 \mathrm{~min}$ at room temperature to recover and then kept in an incubator at $28^{\circ} \mathrm{C}$ on a $16 \mathrm{~h}: 8 \mathrm{~h}$ light/dark cycle. To determine knockdown efficiency, qPCR was performed 3 days post-injection.

\section{Heartbeat Assay: In vivo}

The heartbeat of adult $R$. prolixus injected with either dsCRZR or dsARG was measured 3 days after injection. Animals were immobilized on a Dental wax-coated dissecting dish ventral-side down. Their wings were cut to expose the transparent dorsal cuticle and the in vivo heartbeat was counted by eye per minute under the microscope.

\section{Heartbeat Assay: In vitro}

Adult $R$. prolixus injected 3 days earlier with either dsCRZR or dsARG were dissected from the ventral surface under physiological saline $\left(150 \mathrm{mM} \mathrm{NaCl}, 8.6 \mathrm{mM} \mathrm{KCl}, 2 \mathrm{mM} \mathrm{CaCl}_{2}\right.$, $4 \mathrm{mM} \mathrm{NaHCO}_{3}$, and $8.5 \mathrm{mM} \mathrm{MgCl}_{2}, 5 \mathrm{mM}$ HEPES, $\mathrm{pH}$ 7.0). The digestive and reproductive systems were removed to expose the dorsal vessel. Electrodes attached to an impedance converter (UFI model 2991, Morro Bay, CA, USA) were positioned on either side of the dorsal vessel between the sixth and fifth abdominal segments. The heartbeat frequency was left to stabilize in $50 \mu \mathrm{L}$ saline and the frequency of heartbeat measured from the traces recorded on a linear Flat-bed single channel chart recorder. The saline was then exchanged for $50 \mu \mathrm{L}$ of $10^{-8} \mathrm{M} \mathrm{CRZ}$ and the heartbeat frequency was again measured. The response to $10^{-8}$ M CRZ was quantified by measuring the increase in heartbeat frequency compared to saline control.

\section{Effect of Rhopr-CRZR on Ecdysis and Cuticle Coloration}

Fourth instar $R$. prolixus were injected with $1 \mu \mathrm{L}$ of $2 \mu \mathrm{g} / \mu \mathrm{L}$ dsARG or dsCRZR 10 days post feeding, and kept in an incubator at $28^{\circ} \mathrm{C}$ on a $16 \mathrm{~h}: 8 \mathrm{~h}$ light/dark cycle. The timing of ecdysis and the coloration of the cuticle post-ecdysis were monitored daily.

\section{RESULTS}

\section{Rhopr-CRZR Analysis}

The complete cDNA sequence encoding Rhopr-CRZR was isolated, revealing two splice variants (Rhopr-CRZR- $\alpha$ and $\beta$ ). These encode receptors that are 441 amino acids (RhoprCRZR- $\alpha$, accession number: KU052880) (Figure 1A) and 420 amino acids (Rhopr-CRZR- $\beta$, accession number: KU052881) (Supplementary Figure 1) in length. Rhopr-CRZR- $\alpha$ has seven exons and six introns. Rhopr-CRZR- $\beta$ is missing four nucleotides at the end of exon five, resulting in a frame-shift, causing a truncated ORF (Figure 1B). The CRZR is predicted to have seven alpha-helical transmembrane segments in the ORF as well as three intra- and three extra-cellular loops (Figure 2). The Nterminal domain is on the extracellular side and the C-terminal domain is on the intracellular side. Both transcripts have two putative $\mathrm{N}$-linked glycosylation sites in their $\mathrm{N}$-terminus as well as predicted phosphorylation sites in their intracellular domains. Moreover, two cysteine residues on the first and second extracellular loops are present (Figure 2).

Sequence analysis of Rhopr-CRZR revealed characteristics of a rhodopsin-like GPCR. A divergent Asp-Arg-Phe (DRF) amino acid sequence instead of the Asp-Arg-Tyr (DRY) motif is found at the cytoplasmic end of the third transmembrane domain (Figure 2). Another characteristic of a rhodopsin-like GPCR is the presence of an NSxxNPxxY motif in the seventh transmembrane domain which is also found in the RhoprCRZR (Figure 2). The multiple sequence alignment of RhoprCRZR with other cloned insect CRZRs shows that the protein is highly conserved, mainly over the region comprising the seven 
A

ATG CAG ACG TTA TTT CCA AAC ATT AGT GAC GAA ACT ACG TTA CGC CAA CTT CAA GAC CAT CTG ATT AAT TCA GAT 75 MET Gln Thr Leu phe Pro Asn lle Ser Asp Glu Thr Thr Leu Arg Gln Leu Gln Asp His Leu lle Asn Ser Asp 25 GAC GGT CAT CGC TTा ATA CTG CCT CTA GAA TTA TGT GAT CTC TGG AAT ATA ACA GTC AGT AGT TCG TCG CGT ATA 150 Asp Gly His Arg Phe lle Leu Pro Leu Glu Leu Cys Asp Leu Trp Asn lle Thr Val Ser Ser Ser Ser Arg lle 50 CAA TGT TTG GAA CAT GCA CCC CAG TTG ACA TCT TCG GCA AGA ACT CGT GCC ATT GTG CTC GGT GTG ATG GCC GTC 225 Gln Cys Leu Glu His Ala Pro Gln Leu Thr Ser Ser Ala Arg Thr Arg Ala lle Val Leu Gly Val Met Ala Val 75

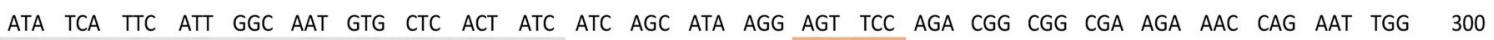
lle Ser Phe lle Gly Asn Val Leu Thr lle lle Ser lle Arg Ser Ser Arg Arg Arg Arg Arg Asn Gln Asn Trp 100 TCG GCG GTA TAC GCT CTG ATC CTC CAT CTC TCA GTG TCT GAT CTC CTG GTC ACA ATA TTC TCG ATA GCT GGA GAA 375 Ser Ala Val Tyr Ala Leu lle Leu His Leu Ser Val Ser Asp Leu Leu Val Thr lle phe Cys lle Ala Gly Glu 125 GCA CTC TGG AGT TAC ACC GTA GCA TGG ACG GCC GAC AAT GTT ACC TCG AAA TTG TTC AAA TTT TCT GAA ATG TTT 450 Ala Leu Trp Ser Tyr Thr Val Ala Trp Thr Ala Asp Asn Val Thr Cys Lys Leu Phe Lys Phe Ser Glu Met Phe 150 GCA CTC TAT CTT TCT ACA TTA ATA TTA GTA TTG ATC GGC CTT GAT CGA TTT GTG GCA GTG CGT TAT CCA ATC AAA 525 Ala Leu Tyr Leu Ser Thr phe lle Leu Val Leu lle Gly Leu Asp Arg phe Val Ala Val Arg Tyr pro lle Lys 175 GTA ATC AGC ACT GCG AAA AGA TGT GGC CGA TाT GTT GCT GGA GCT TGG TTT CTA AGC TाT CTT TTA ATG CTT CCT 600 Ala lle Ser Thr Ala Lys Arg Cys Gly Arg Phe Val Ala Gly Ala Trp Phe Leu Ser Phe Leu Leu Ser Leu Pro 200

CAG GTG TTC ATA TIT CAC CTG TCT AAA GGA CCA TTT TAT GAA GAA TIT TAT CAG TGT GTA ACT TAT GGC TIT TAT 675 Gln Val Phe lle Phe His Leu Ser Lys Gly Pro Phe Tyr Glu Glu Phe Tyr Gln Cys Val Thr Tyr Gly Phe Tyr 225

ACA GAA CCA TGG CAA GAA CAA TTG TAC ACA ACC TTC AGC $\Pi T$ GTG TGT ATG TTC ATG TTA CCA TTA TTG ATT CTA 750 Thr Glu Pro Trp Gln Glu Gln Leu Tyr Thr Thr Phe Ser Phe Val Cys Met Phe Met Leu Pro Leu Leu lle Leu 250

ATC ATT TCG TAT GTG TCA ACA ATA ATC ACA ATT TCA CAA AGT GAC AAA ATG TTC CGT GAT GAA AGT AAC AAT ACT 825 lle lle Ser Tyr Val Ser Thr lle lle Thr lle Ser Gln Ser Asp lys Met phe Arg Asp Glu Ser Asn Asn thr 275

AGT GCT ACA AGA AAA TTG GAC ATT AAT CGA AGA AGA TTA ATA CAC AGA GCA AAG ATG AAA TCA TTT AGA ATA TCT 900 Ser Ala Thr Arg Lys Leu Asp lle Asn Arg Arg Arg Leu lle His Arg Ala Lys Met lys Ser phe Arg lle Ser 300 CTG GTC ATT GTG GTT ACA TTा ATT GTC TGG TGG ACT CCA TAT TAC ACG ATG ATG ATA ATT TTT ATG TTT TTA AAT 975 Leu Val lle Val Val Thr Phe lle Val Trp Trp Thr Pro Tyr Tyr Thr Met Met lle lle Phe Met Phe Leu Asn 325

CCA GAT AAA CAT TTG AGC GAA GAA TTA CAA AAA GGA ATA TTC TTC TTT GGA ATG TCT ACA AGC CTT GTA AAT CCA 1050 Pro Asp Lys His Leu Ser Glu Glu Leu Gln Lys Gly lle phe Phe phe Gly Met Ser Asn Ser Leu Val Asn Pro 350

CTA ATA TAT GGT GCT TTT TAT CTT TGG CGA CCT TCA AAG AAA ACC GGG AGC GCT AGg TCT GGA AGg GAG CAC GCT 1125 Leu Ile Tyr Gly Ala Phe His Leu Trp Arg Pro Ser Lys Lys Thr Gly Ser Ala Arg Ser Gly Arg Glu His Ala 375

ACA TAC TCC TTG CTC AAG AGG ACT CGG ACG TGG CAC ACT CCG GGA GGA AGA GAC TAC CTT TGT CTT AAT AGA ACA 1200 Thr Tyr Ser Leu Leu Lys Arg Thr Arg Thr Trp His Thr Pro Gly Gly Arg Asp Tyr Leu Cys Leu Asn Arg Thr 400

AAC GGC GAC AGC AAA CAA TTा CAA CAG ATC AGC GTC CTT TTG CCA AAC AAC AAG GAT CCA ATA ACA ATT ACC AAT 1275 Asn Gly Asp Ser Lys Gln Phe Gln Gln lle Ser Val Leu Leu Pro Asn Asn Lys Asp Pro lle Thr lle Thr Asn 425 AAA GTA AGC AAA CTT GGG CTA AGC AGA AGT ATG TAC AGG ATA GCA TCG TAA AACATCTGGCAGATGTAATGTTACCTAAT TAATGT 1361 Lys Val Ser lys Leu Gly Leu Ser Arg Ser Met Tyr Arg lle Ala Ser * ATATATTTTTCTTTCTGAAAGAGAGAAAGAAAAAAAAATGCCAAAGAAACTTTAATTTAGGTATATTGTATTTTAAAAGGAAAGGGATCAGCTAGC - 3'

\section{B}

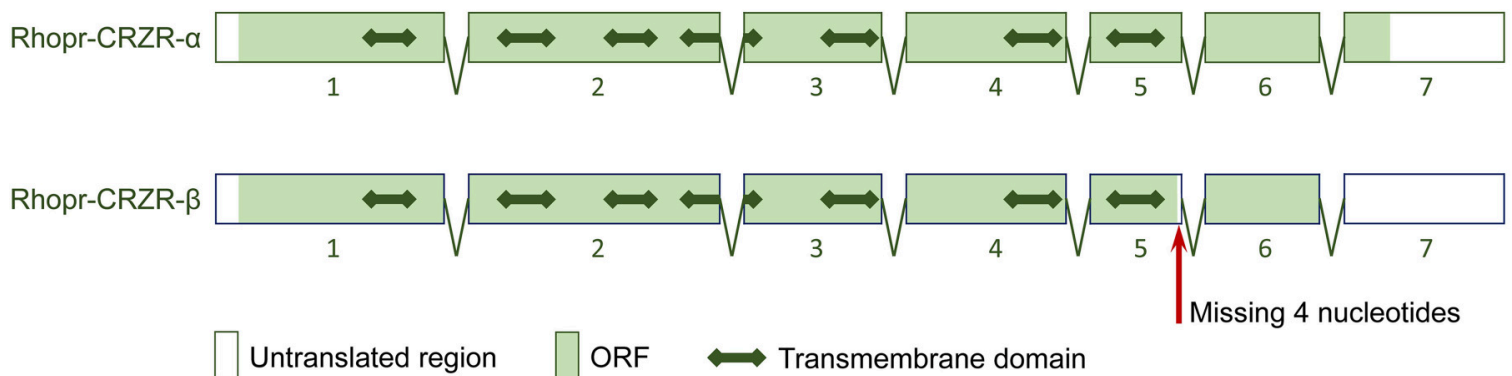

FIGURE 1 | Rhopr-CRZR- $\alpha$ sequence and gene structure. (A) The cDNA sequence and its deduced amino acid sequence of Rhopr-CRZR- $\alpha$. The numbering of the amino acids starts with the first methionine of the ORF. The stop codon upstream of the start codon is underlined. The seven transmembrane helices, the predicted N-linked glycosylation sites and predicted phosphorylation sites are shaded in gray, blue and orange, respectively. (B) The splicing pattern of Rhopr-CRZR- $\alpha$ and $-\beta$ as predicted using BLAST and splice site prediction software. The boxes represent exons, and the two-headed arrow represents the transmembrane domains. 




transmembrane domains and the conserved regions specific for rhodopsin-like GPCRs (Supplementary Figure 2).

\section{Functional Characterization of Rhopr-CRZR}

To determine specificity of the cloned Rhopr-CRZR to its putative ligand, Rhopr-CRZ, a calcium mobilizing assay was used where both receptor transcripts were separately transiently transfected into CHOKI-aeq cells. Rhopr-CRZR- $\alpha$ and $\beta$ were dose-dependently activated by Rhopr-CRZ with an $\mathrm{EC}_{50}$ value of 2.7 and $1 \mathrm{nM}$, respectively (Figure 3A). Maximum luminescence was seen during the first $5 \mathrm{~s}$ following activation of either receptor with Rhopr-CRZ (Figures 3B,C). Neither receptor was activated by the evolutionarily-related peptides, Rhopr-AKH, or RhoprACP (Figures 3B,C), which contain some sequence similarity (Table 1). Moreover, control cells transfected with an empty vector did not elicit a response.

\section{Transcript Expression Profile of Rhopr-CRZR}

The spatial expression pattern of the Rhopr-CRZR transcript was used to determine which peripheral tissues express the receptor to give clues on the functional roles for the RhoprCRZ signaling pathway. In 5 th instar $R$. prolixus, the highest transcript expression was found in the CNS (Figure 4). Similar expression levels were found in the different parts of the CNS; the brain and suboesophageal ganglion (Brain), the prothoracic ganglion (PRO), and mesothoracic ganglionic mass (MTGM) (Figure 4 inset). Cells that express the Rhopr-CRZR mRNA were localized in the brain using fluorescent in situ hybridization (FISH) (Figure 5). On the dorsal side of the brain, a group of 7 bilaterally paired medial cells were observed, two of which are smaller in diameter than the other 5. On the basis of size, shape and location, these appear to be a subset of the medial neurosecretory cells.

The spatial expression pattern also revealed high expression in the dorsal vessel, similar to that of the CNS (Figure 4). The receptor transcript was also expressed at moderate levels in the abdominal dorsal epidermis, and prothoracic glands with associated fat body. There was minimal transcript expression in the foregut, midgut, and hindgut. Interestingly, transcript expression was also found in the female and male reproductive tissues (Figure 4).

\section{Rhopr-CRZR Role in Regulating Heartbeat Rate}

The percent knockdown of Rhopr-CRZR transcript after injection of dsCRZR into adult $R$. prolixus was quantified by qPCR in the dorsal vessel. It was found to be knocked down by $86.9 \pm 2.9 \%$ relative to control dsARG injected insects 3 days after injection. The physiological effects of this decrease in RhoprCRZR on heartbeat frequency was studied in vivo and in vitro in adult $R$. prolixus. In vivo, the heartbeat frequency of insects previously injected with dsCRZR (42 \pm 1.63 beats/min, $\mathrm{n}=5)$ 
A

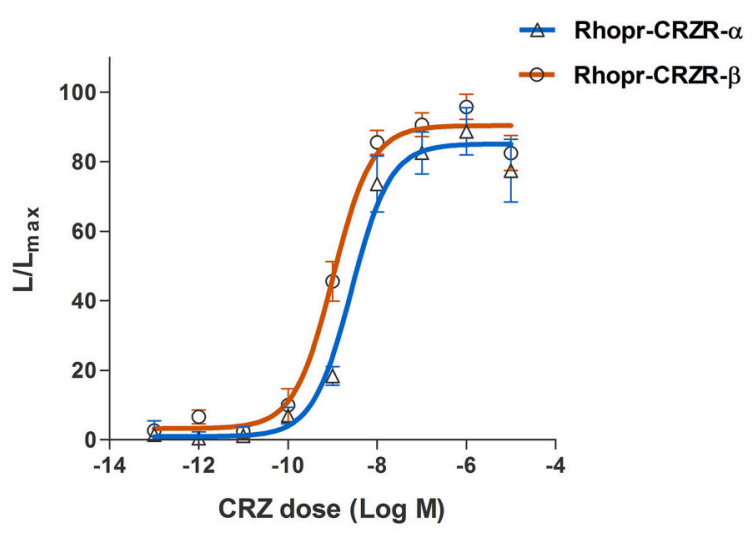

\begin{tabular}{ll} 
Rhopr-CRZR- $\alpha$ EC $_{50}$ & $2.7 \mathrm{nM}$ \\
\hline Rhopr-CRZR- $\beta$ EC $_{50}$ & $1 \mathrm{nM}$
\end{tabular}

B

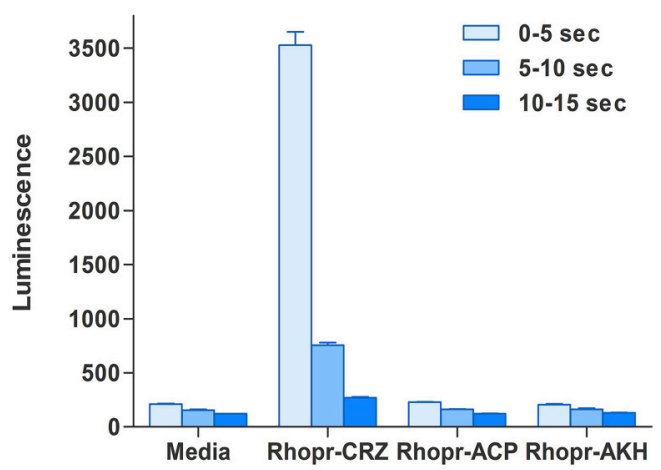

C

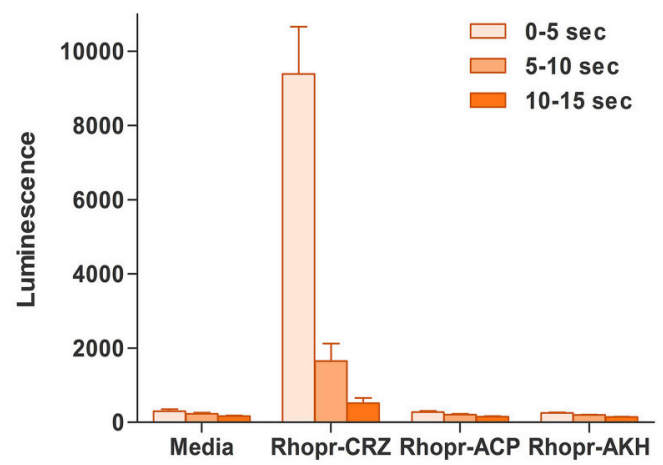

FIGURE 3 | Functional receptor assay of Rhopr-CRZR- $\alpha$ and $\beta$ transiently expressed in CHOK1-aeq cells. (A) Dose-response curve of the luminescence response following the addition of Rhopr-CRZ to cells expressing Rhopr-CRZR- $\alpha$ or $\beta$. The EC 50 values of Rhopr-CRZR- $\alpha$ and $\beta$ are 2.7 and $1 \mathrm{nM}$, respectively. Kinetics of the bioluminescence response of Rhopr-CRZR- $\alpha$ (B) and Rhopr-CRZR- $\beta$ (C) recorded every $5 \mathrm{~s}$ over a $15 \mathrm{~s}$ interval following the addition of media, Rhopr-CRZ, Rhopr-ACP, and Rhopr-AKH at a dose of $10^{-6} \mathrm{M}$. Rhopr-ACP and Rhopr-AKH were without effect on these receptors. Values represent mean \pm error of mean $(n=3)$.

was decreased by $13.6 \%$ compared to control insects injected with dsARG $(48.6 \pm 1.4$ beats/min, $n=5$ ) (unpaired $t$-test, $p=0.0188$ ) (Figure 6A). Previously, Rhopr-CRZ has been shown to increase heartbeat frequency in a dose-dependent manner in vitro in 5 th instar R. prolixus (Patel et al., 2014). To verify the in vivo results, the heartbeat frequency was investigated in vitro to determine if the reduced heartbeat frequency in vivo was due to the decrease in Rhopr-CRZR. After the addition of $10^{-8} \mathrm{M}$ Rhopr-CRZ, the heartbeat frequency of dsARG injected insects increased by 7.33 \pm 0.8 beats/min $(n=5)$ whereas the heartbeat frequency only increased by $2 \pm 0.9$ beats $/ \min (n=5)$ in dsCRZR injected insects (unpaired $t$-test, $p=0.0096$ ) (Figure 6B).

\section{The Possible Role of Rhopr-CRZ in Regulating Ecdysis}

The possible involvement of the Rhopr-CRZ-signaling pathway on ecdysis and cuticle coloration was monitored in 4th instar to 5 th instar $R$. prolixus. Under the specific experimental conditions, ecdysis took place 11-16 days post-feeding of 4 th instars on
TABLE 1 | Amino acid sequences and similarities between Rhopr-CRZ, Rhopr-ACP, and Rhopr-AKH in R. prolixus.

\begin{tabular}{llllllllllllll}
\hline Neuropeptide & \multicolumn{10}{c}{ Amino acid sequence } \\
\hline Rhopr-CRZ & $\mathrm{PQ}$ & - & $\mathrm{T}$ & $\mathrm{F}$ & $\mathrm{Q}$ & $\mathrm{Y}$ & $\mathrm{S}$ & $\mathrm{R}$ & $\mathrm{G}$ & $\mathrm{W}$ & $\mathrm{T}$ & $\mathrm{N}$ & amide \\
Rhopr-ACP & $\mathrm{PQ}$ & $\mathrm{V}$ & $\mathrm{T}$ & $\mathrm{F}$ & - & - & $\mathrm{S}$ & $\mathrm{R}$ & $\mathrm{D}$ & $\mathrm{W}$ & $\mathrm{N}$ & $\mathrm{A}$ & amide \\
Rhopr-AKH & $\mathrm{PQ}$ & $\mathrm{L}$ & $\mathrm{T}$ & $\mathrm{F}$ & - & - & $\mathrm{S}$ & $\mathrm{T}$ & $\mathrm{D}$ & $\mathrm{W}$ & - & - & amide
\end{tabular}

rabbit's blood (Figure 7A). Insects injected with dsARG $(n=21)$ and dsCRZR $(n=20)$ displayed no significant difference in ecdysis behavior or timing of ecdysis (Figure 7A); however, the peak of ecdysis for the dsARG injected insects was 13 days postfeeding compared to 12 days post-feeding for dsCRZR injected insects. Perhaps this might be caused by decreasing heartbeat frequency and influencing hemolymph circulation. The percent knockdown of the Rhopr-CRZR transcript in the CNS was $79 \pm$ $1 \%$ relative to dsARG injected bugs 3 days after injections. The transcript levels of Rhopr-CRZR at different time points around 


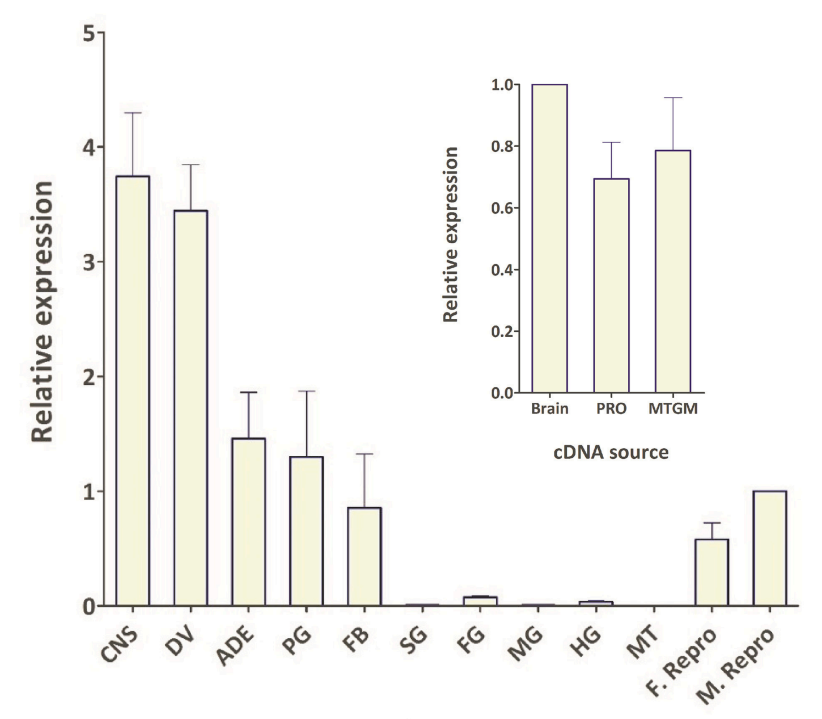

cDNA source

FIGURE 4 | Spatial expression pattern of Rhopr-CRZR transcript in 5th instar $\boldsymbol{R}$. prolixus using qPCR. The expression was analyzed in the central nervous system (CNS), dorsal vessel (DV), abdominal dorsal epidermis (ADE), prothoracic glands and its associated fat body (PG), fat body (FB), salivary glands (SG), foregut (FG), midgut (MG), hindgut (HG), Malpighian tubules (MT), female reproductive system (F. Repro), and male reproductive system (M. Repro). Fold difference is relative to transcript levels in M. Repro cDNA. Inset shows the expression in the brain plus suboesophaegeal ganglion (Brain), prothoracic ganglion (PRO), and mesothoracic ganglionic mass (MTGM). Fold difference is relative to transcript levels in the brain CDNA. The GPCR was performed on three biological replicates with three technical replicates each. Values represent mean \pm error of mean.
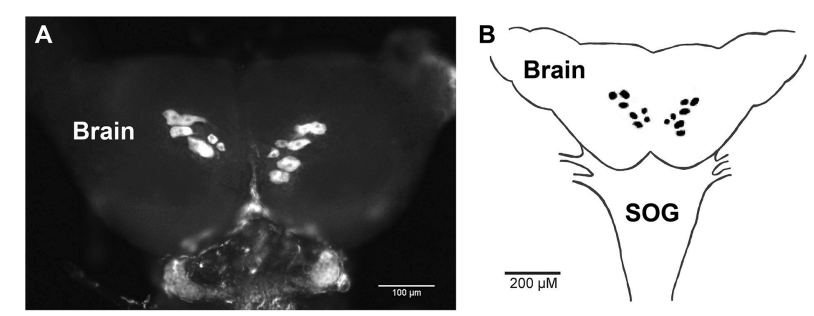

FIGURE 5 | Fluorescent in situ hybridization of Rhopr-CRZR transcript in the brain of 5th instar R. prolixus. (A) Confocal microscopy image of the brain revealing a group of seven bilaterally paired medial cells on the dorsal surface. (B) Diagram illustrating the location of cells in the brain containing the Rhopr-CRZR transcript. The fluorescent in situ hybridization was performed three times with eight CNSs each time. SOG, suboesophageal ganglion.

ecdysis were measured using qPCR. There was no significant difference in the expression of Rhopr-CRZR from 3 days preecdysis to 3 days post-ecdysis (Figure 7B).

\section{The Possible Role of Rhopr-CRZR in Regulating Cuticle Coloration}

Following ecdysis, the coloration of the cuticle was monitored to determine the effect of decreases in Rhopr-CRZR levels on cuticle

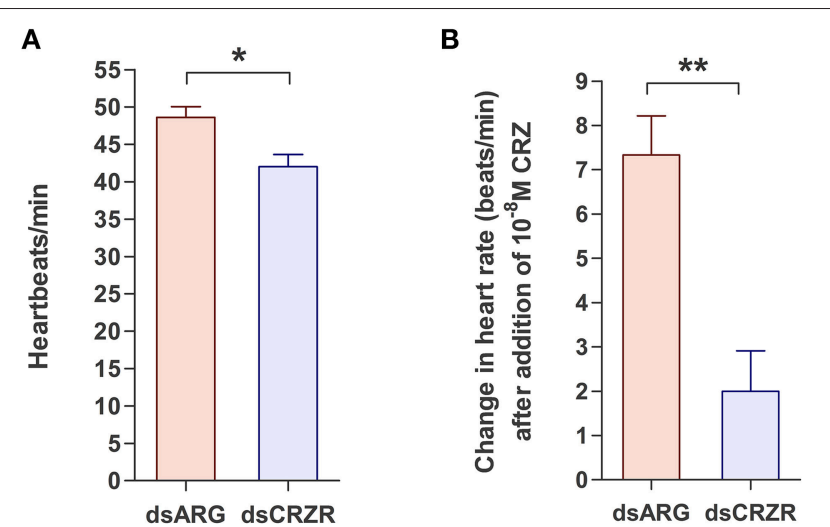

FIGURE 6 | The effect of Rhopr-CRZR on heartbeat in adult $\boldsymbol{R}$. prolixus. $2 \mu \mathrm{g}$ of dsARG or dsCRZR was injected into adult $R$. prolixus and the effect on heartbeat was measured 3 days post injection. Values represent mean \pm error of mean $(n=5)$. (A) Heartbeat frequency in vivo in dsARG and dsCRZR injected insects. There is a significant decrease in heartbeats per minute in dsCRZR injected insects (unpaired $t$-test, ${ }^{*} p=$ 0.0188). (B) The effect of $10^{-8} \mathrm{M}$ Rhopr-CRZ in vitro on heartbeat rate in dsARG and dsCRZR injected insects. Rhopr-CRZ at $10^{-8} \mathrm{M}$ significantly increased the heartbeat rate in dsARG injected insects but not in dsCRZR injected insects (unpaired $t$-test, ${ }^{* *} p=0.0096$ ).

coloration. Insects injected with dsARG $(n=21)$ and dsCRZR $(n=20)$ did not show any difference in coloration or the time it takes to darken the cuticle (Figure 8).

\section{DISCUSSION}

In this study, we isolated and characterized the cDNA sequence encoding Rhopr-CRZR. The CRZR is present in a number of insects such as A. gambiae, B. mori, D. melanogaster, M. sexta, and $M$. domestica. Their sequences are highly conserved and similar to the CRZR sequence isolated and cloned in $R$. prolixus. Two CRZRs have been found in $R$. prolixus, whereas only one CRZR has been identified in other insects. The two receptors are a result of alternative splicing causing one receptor (RhoprCRZR- $\alpha$ ) to have a larger C-terminal than the other (RhoprCRZR- $\beta$ ). Alternative splicing causes structural diversity where a single transcript can generate more than one mRNA (Journot et al., 1994). This phenomenon has been selected in evolution as it can regulate or modify the intensity and specificity of the binding of proteins to the receptor (Journot et al., 1994).

Specificity of the Rhopr-CRZR was determined using CHOK1-aeq cells where only Rhopr-CRZ elicited a response, confirming ligand binding. Through gene duplication, and successive specialization, the CRZ, AKH, and ACP-signaling pathways emerged (Hauser and Grimmelikhuijzen, 2014). To deduce the specificity of the Rhopr-CRZR to RhoprCRZ, Rhopr-AKH, and Rhopr-ACP were tested to examine if they elicited a response in the functional receptor assay, and they did not. Despite the sequence similarity between these neuropeptides, there is no cross-reactivity between the 


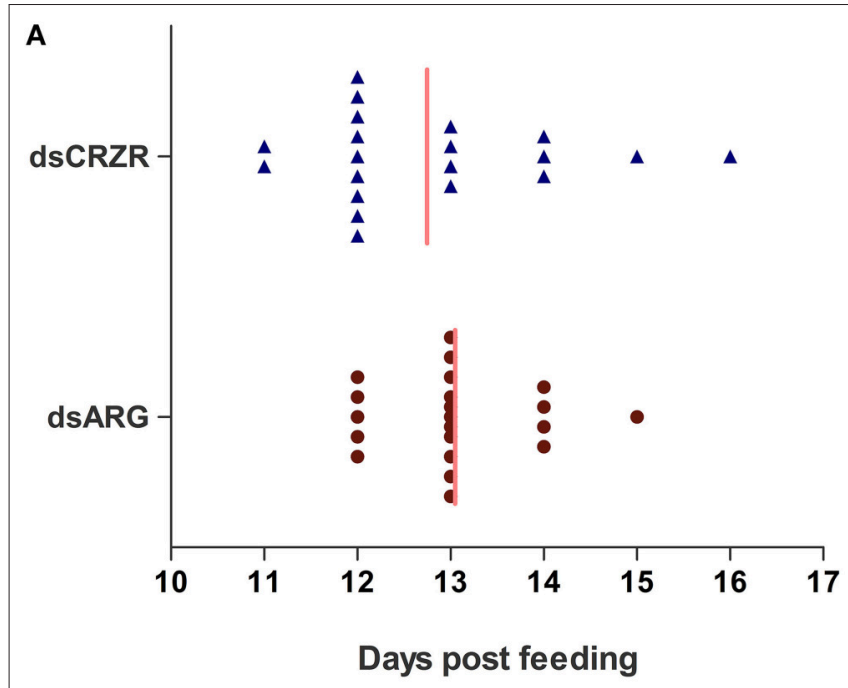

B

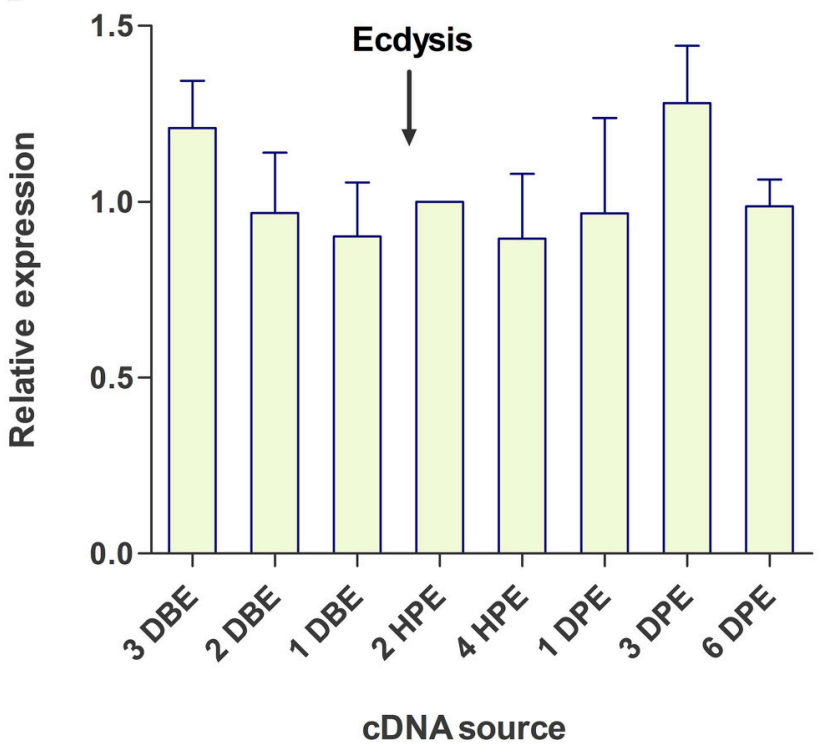

FIGURE 7 | The effect of Rhopr-CRZR on ecdysis in R. prolixus. (A) 4th instar $R$. prolixus were injected with $2 \mu \mathrm{g}$ of dsARG or dsCRZR 10 days post feeding. Ecdysis took place 11-16 days post-feeding. There was no significant effect on the timing of ecdysis between dsARG RNA and dsCRZR injected insects; however, dsCRZR injected insects were observed to undergo ecdysis a day earlier causing a shift in the median (vertical line). (B) Temporal expression analysis of Rhopr-CRZR transcript in the CNS by qPCR indicated that the receptor expression is not up-regulated around the timing of ecdysis. The expression was analyzed in 4th instar CNS 3 days before ecdysis (DBE), 2 DBE, 1 DBE, $2 \mathrm{~h}$ post-ecdysis (HPE) into 5th instar, $4 \mathrm{HPE}, 1$ day post-ecdysis (DPE), 3 DPE, and 6 DPE. Fold difference is relative to transcript levels of 2 HPE. The experiment was repeated on three biological replicates with three technical replicates each. Values represent mean \pm error of mean.

different signaling pathways (Hansen et al., 2010; Patel et al., 2014).

As a preliminary investigation into possible roles of RhoprCRZ in the CNS we performed in situ hybridization on the brain of 5th instars. Interestingly, seven bilaterally-paired median

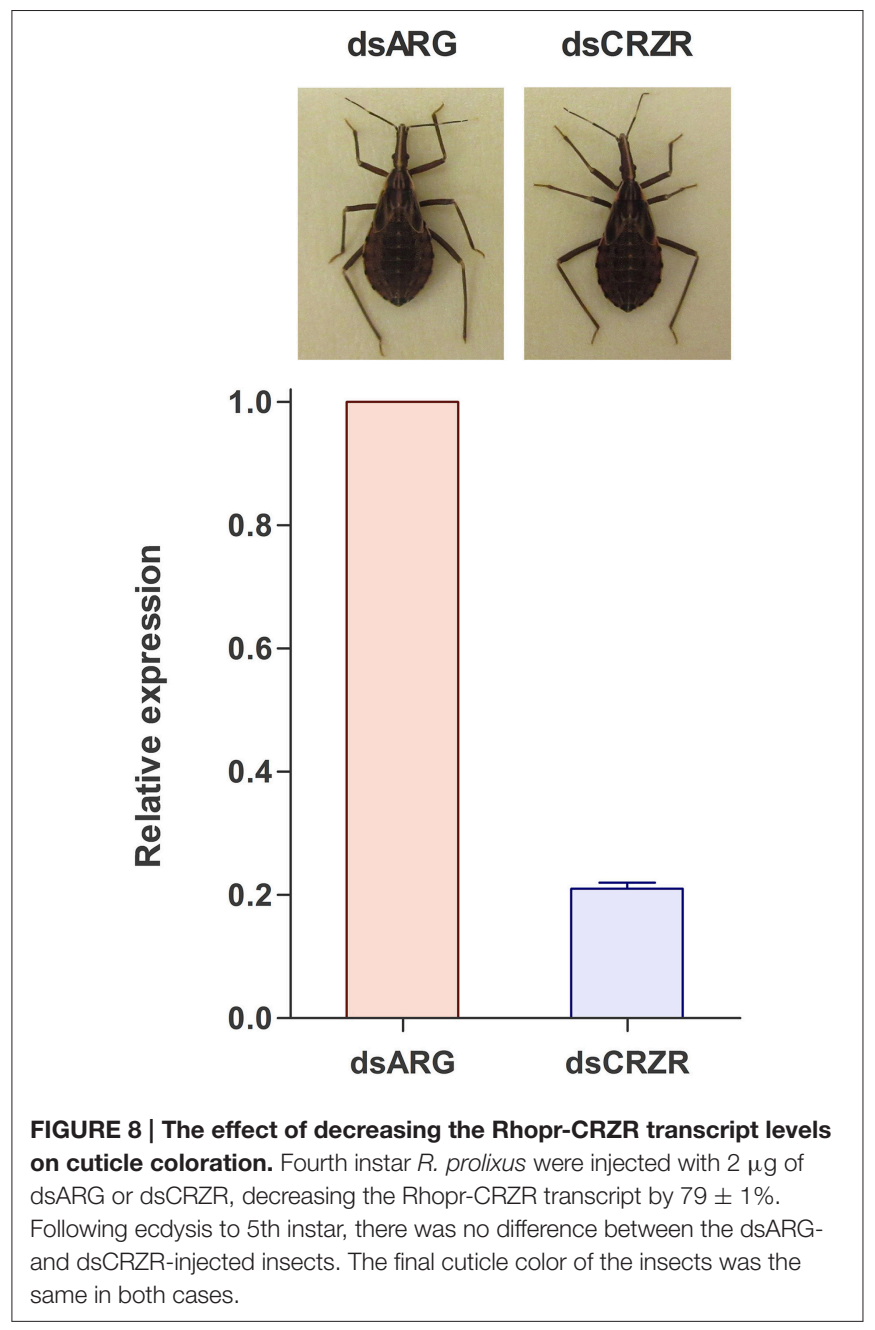

cells express the receptor transcript. These cells are of a size and location of medial neurosecretory cells, and so Rhopr-CRZ may be involved in coordinating neuroendocrine activities in $R$. prolixus. Similar CRZR transcript expression in a subset of medial neurosecretory cells has also been shown in Drosophila (Zandawala personal communication). Clearly, additional in situ hybridization studies will be required for all tissues over a developmental cycle to gain further insight into the CRZsignaling pathways.

Quantitative analysis of the Rhopr-CRZR transcript expression can also reveal the peripheral tissues that express the receptor, giving clues for functional roles. The presence of the receptor implies that CRZ should influence the activity of that tissue. Rhopr-CRZR transcript expression was seen throughout the CNS and associated with peripheral tissues. These included the dorsal vessel, abdominal dorsal epidermis, prothoracic glands and associated fat body, abdominal fat body, and male and female reproductive tissue.

High expression of Rhopr-CRZR transcript in the dorsal vessel was not unexpected since Rhopr-CRZ stimulates heartbeat frequency in $R$. prolixus (Patel et al., 2014). To further confirm 
the role of the CRZ-signaling pathway on the dorsal vessel in $R$. prolixus, the Rhopr-CRZR transcript was knocked down using dsRNA. Significantly lowering the transcript levels in adults led to a decrease in heartbeat frequency in vivo indicating that CRZ is involved in the regulation of heartbeat in the intact animal. Moreover, Rhopr-CRZR knockdown significantly reduced the effect of Rhopr-CRZ on heartbeat frequency in vitro. Thus, Rhopr-CRZ was less effective at increasing heartbeat rate in vitro in insects which had knockdown of the Rhopr-CRZR transcript. It has been previously shown in $R$. prolixus that $\mathrm{CRZ}$ increases the heartbeat frequency in a dose-dependent manner (Patel et al., 2014). This is the first report to show that the CRZ-signaling pathway is involved in the regulation of heartbeat frequency in intact adult R. prolixus.

The expression of the Rhopr-CRZR in the prothoracic glands implies that the CRZ-signaling pathway may play a role in the molt cycle since the prothoracic glands is essential for releasing ecdysteroids that regulate molting. Wigglesworth (1934) found that following a blood meal, a hormone which was later termed the prothoracictropic hormone (PTTH), acted on the prothoracic glands, triggering the secretion of ecdysteroids into the hemolymph (Kataoka et al., 1991). These ecdysteroids coordinate molting by regulating the expression of specific neuropeptide genes coding for, for example, PETH and ETH (Žitňan et al., 1996, 1999). To determine if Rhopr-CRZ also initiates ecdysis in $R$. prolixus, similar to that found in M. sexta, the Rhopr-CRZR was knocked down prior to ecdysis; however, data showed that it did not have an effect on timing or duration of ecdysis under the experimental protocol used here. Moreover, receptor transcript levels were not upregulated in the CNS around the time of ecdysis, again indicating that the RhoprCRZR transcript levels were not influenced during the timing of ecdysis or behaviors associated with ecdysis. The CRZ-signaling pathway may not be involved in ecdysis, but perhaps involved earlier in the molting process. For example, it could play a role with PTTH in regulating production and release of ecdysteroids, implying that PTTH may not act alone and possibly the ingestion of a large blood meal may also trigger the release of CRZ to act on the prothoracic glands, also triggering the release of ecdysteroids.

Expression of Rhopr-CRZR transcript was observed in the abdominal dorsal epidermis suggesting the involvement of the corazonin-signaling pathway in cuticle coloration as found in locusts (Tawfik et al., 1999). In $R$. prolixus, following ecdysis, the newly molted insect has a soft, and light colored cuticle that hardens and darkens in the subsequent hours postecdysis. The effect of Rhopr-CRZ on the coloration of the cuticle was examined by decreasing the RhoprCRZR transcript levels using RNAi. Following ecdysis, both the control and experimental insects were the same color and darkened at the same rate resulting in identical cuticle colors. Therefore, despite a significant knockdown of the transcript, there was no effect on coloration. Even though the CRZ peptide was found to induce tanning of the cuticle in locusts (Tawfik et al., 1999; Tanaka, 2000), it did not have that effect on other insects such as G. mellonella, G. bimaculatus, and B. mori (Hua et al., 2000;
Hansen et al., 2001), and may also not be involved in this in R. prolixus.

Perhaps the expression of the Rhopr-CRZR transcript in the anterior dorsal epidermis is due to its involvement in other functions such as cuticle secretion. During ecdysis, the epidermal cells elongate, separating themselves from the old cuticle. Before molting to the next instar, a new cuticle is set down by first secreting the epicuticle, the exocuticle and finally the endocuticle. The Rhopr-CRZ pathway could be involved in the regulation of this process.

It was interesting and unexpected to find expression of RhoprCRZR transcript associated with the reproductive system since reproductive functions for the $\mathrm{CRZ}$-signaling pathway in insects have not been reported. CRZ is believed to have a vertebrate homolog, GnRH (Hauser and Grimmelikhuijzen, 2014; see Roch et al., 2014) and so the CRZ-signaling pathway may have reproduction functions similar to $\mathrm{GnRH}$. GnRH is a key regulator of reproductive maturation in vertebrates and recently GnRH-type and CRZ-type signaling systems were identified in echinoderms illustrating the paralogous CRZ-signaling pathway is present in the common ancestor of the Bilateria (Semmens et al., 2016).

Despite CRZ having a conserved structure throughout evolution, it does not have a conserved function - from affecting cardiovascular activity in some insects to affecting ecdysis and cuticle coloration in others. Perhaps these functions are not distinct and fall under the common factor of regulating physiological stress (Boerjan et al., 2010). Examples of stressors that threaten homeostasis include extreme temperature, humidity, food shortage, light intensity, and excessive population density. Cardiovascular activity can be induced by a number of physiological stressors. For example, after the ingestion of a large blood meal in $R$. prolixus, the contractions of the anterior midgut push the hemolymph to the posterior end of the insect. To help circulate the hemolymph throughout the bug, four pairs of ostia or valves that make up the heart, open, taking in hemolymph which is propelled anteriorly through the dorsal vessel by heart muscle contractions (Chiang et al., 1990). CRZ may increase heartbeat frequency in $R$. prolixus in order to accelerate hemolymph circulation during times of stress (e.g., massive blood meal). Having a cardioacceleratory effect also results in Rhopr-CRZ itself being circulated. Cuticle darkening in locusts is thought to be a result of different stressors such as food deprivation (Veenstra, 2009), and the involvement of CRZ in ecdysis initiation in $M$. sexta may be due to a response to stress as ecdysis is a critical part of development that is dependent on nutrient and water balance (Zhao et al., 2010). Further research may uncover additional roles of CRZ, enhancing our understanding of the role that CRZ plays in various behaviors.

\section{AUTHOR CONTRIBUTIONS}

$\mathrm{ZH}$, Designed and performed all experiments, data analysis and written work. AL, Contributed to experimental design and revisions to manuscript. IO, Contributed to experimental design and revisions to manuscript. 


\section{FUNDING}

This work has been funded by the Natural Sciences and Engineering Research Council of Canada (NSERC) to AL (RGPIN 2014-06253) and IO (RGPIN 8522-12).

\section{ACKNOWLEDGMENTS}

We are grateful to Jean-Paul Paluzzi (York University, Canada) for initiating early aspects of this research, NSERC for

\section{REFERENCES}

Ballesteros, J. A., Shi, L., and Javitch, J. A. (2001). Structural mimicry in G proteincoupled receptors: implications of the high-resolution structure of rhodopsin for structure-function analysis of rhodopsin-like receptors. Mol. Pharmacol. 60, 1-19. doi: 10.1124/mol.60.1.1

Belmont, M., Cazzamali, G., Williamson, M., Hauser, F., and Grimmelikhuijzen, C. J. (2006). Identification of four evolutionarily related G protein-coupled receptors from the malaria mosquito Anopheles gambiae. Biochem. Biophys. Res. Commun. 344, 160-165. doi: 10.1016/j.bbrc.2006.03.117

Boerjan, B., Verleyen, P., Huybrechts, J., Schoofs, L., and De Loof, A. (2010). In search for a common denominator for the diverse functions of arthropod corazonin: a role in the physiology of stress? Gen. Comp. Endocrinol. 166, 222-233. doi: 10.1016/j.ygcen.2009.09.004

Caers, J., Verlinden, H., Zels, S., Vandersmissen, H. P., Vuerinckx, K., and Schoofs, L. (2012). More than two decades of research on insect neuropeptide GPCRs: an overview. Front Endocrinol. 3:151. doi: 10.3389/fendo.2012.00151

Cazzamali, G., Saxild, N., and Grimmelikhuijzen, C. (2002). Molecular cloning and functional expression of a Drosophila corazonin receptor. Biochem. Biophys. Res. Commun. 298, 31-36. doi: 10.1016/S0006-291X(02)02398-7

Chiang, R. G., Chiang, J. A., and Davey, K. G. (1990). Morphology of the dorsal vessel in the abdomen of the blood-feeding insect Rhodnius prolixus. J. Morphol. 204, 9-23. doi: 10.1002/jmor.1052040103

Defferrari, M. S., Orchard, I., and Lange, A. B. (2016). Identification of the first insulin-like peptide in the disease vector Rhodnius prolixus: involvement in metabolic homeostasis of lipids and carbohydrates. Insect Biochem. Mol. Biol. 70, 148-159. doi: 10.1016/j.ibmb.2015.12.009

Fredriksson, R., Lagerstrom, M. C., Lundin, L. G., and Schiöth, H. B. (2003). The G-protein-coupled receptors in the human genome form five main families. Phylogenetic analysis, paralogon groups, and fingerprints. Mol. Pharmacol. 63, 1256-1272. doi: 10.1124/mol.63.6.1256

Hansen, I. A., Sehnal, F., Meyer, S. R., and Scheller, K. (2001). Corazonin gene expression in the waxmoth Galleria mellonella. Insect Mol. Biol. 10, 341-346. doi: 10.1046/j.0962-1075.2001.00272.x

Hansen, K. K., Stafflinger, E., Schneider, M., Hauser, F., Cazzamali, G., Williamson, M., et al. (2010). Discovery of a novel insect neuropeptide signaling system closely related to the insect adipokinetic hormone and corazonin hormonal systems. J. Biol. Chem. 285, 10736-10747. doi: 10.1074/jbc.M109.045369

Hauser, F., and Grimmelikhuijzen, C. J. (2014). Evolution of the $\mathrm{AKH} /$ corazonin/ACP/GnRH receptor superfamily and their ligands in the Protostomia. Gen. Comp. Endocrinol. 209, 35-49. doi: 10.1016/j.ygcen.2014.07.009

Hillyer, J. F., Estévez-Lao, T. Y., Funkhouser, L. J., and Aluoch, V. A. (2012). Anopheles gambiae corazonin. Gene structure, expression and effect on mosquito heart physiology. Insect Mol. Biol. 21, 343-355. doi: 10.1111/j.13652583.2012.01140.x

Hua, Y., Ishibashi, J., Saito, H., Tawfik, A., Sakakibara, M., Tanaka, Y., et al. (2000). Identification of [Arg7] corazonin in the silkworm, Bombyx mori and the cricket, Gryllus bimaculatus, as a factor inducing dark color in an albino strain of the locust, Locusta migratoria. J. Insect Physiol. 46, 853-859. doi: 10.1016/S0022-1910(99)00173-0

Journot, L., Spengler, D., Pantaloni, C., Dumuis, A., Sebben, M., and Bockaert, J. (1994). The PACAP receptor: generation by alternative splicing of functional funding, and Meet Zandawala (University of Stockholm, Sweden) for permission to reference data as personal communication.

\section{SUPPLEMENTARY MATERIAL}

The Supplementary Material for this article can be found online at: http://journal.frontiersin.org/article/10.3389/fnins. 2016.00357 diversity among G protein-coupled receptors in nerve cells. Semin. Cell Biol. 5, 263-272. doi: 10.1006/scel.1994.1032

Kataoka, H., Nagasawa, H., Isogai, A., Ishizaki, H., and Suzuki, A. (1991). Prothoracicotropic hormone of the silkworm, Bombyx mori: amino acid sequence and dimeric structure. Agric. Biol. Chem. 55, 73-86.

Kim, Y. J., Spalovská-Valachová, I., Cho, K. H., Zitnanova, I., Park, Y., Adams, M. E., et al. (2004). Corazonin receptor signaling in ecdysis initiation. Proc. Natl. Acad. Sci. U.S.A. 101, 6704-6709. doi: 10.1073/pnas.03052 91101

Mesquita, R. D., Vionette-Amaral, R. J., Lowenberger, C., Rivera-Pomar, R., Monteiro, F. A., Minx, P., et al. (2015). Genome of Rhodnius prolixus, an insect vector of Chagas disease, reveals unique adaptations to hematophagy and parasite infection. Proc. Natl. Acad. Sci. U.S.A. 112, 14936-14941. doi: 10.1073/pnas.1506226112

Oldham, W. M., and Hamm, H. E. (2008). Heterotrimeric G protein activation by G-protein-coupled receptors. Nat. Rev. Mol. Cell Biol. 9, 60-71. doi: $10.1038 / \mathrm{nrm} 2299$

Orchard, I., Lange, A. B., and Bendena, W. G. (2001). FMRFamide-related peptides: a multifunctional family of structurally related neuropeptides in insects. Adv. Insect Physiol. 28, 267-329. doi: 10.1016/S0065-2806(01) 28012-6

Paluzzi, J. P., and O’Donnell, M. J. (2012). Identification, spatial expression analysis and functional characterization of a pyrokinin-1 receptor in the Chagas' disease vector, Rhodnius prolixus. Mol. Cell Endocrinol. 363, 36-45. doi: 10.1016/j.mce.2012.07.007

Paluzzi, J. P., Park, Y., Nachman, R. J., and Orchard, I. (2010). Isolation, expression analysis, and functional characterization of the first antidiuretic hormone receptor in insects. Proc. Natl. Acad. Sci. U.S.A. 107, 10290-10295. doi: 10.1073/pnas.1003666107

Paluzzi, J. P., Russell, W. K., Nachman, R. J., and Orchard, I. (2008). Isolation, cloning, and expression mapping of a gene encoding an antidiuretic hormone and other CAPA-related peptides in the disease vector, Rhodnius prolixus. Endocrinology. 149, 4638-4646. doi: 10.1210/en.2008-0353

Park, Y., Kim, Y.-J., and Adams, M. E. (2002). Identification of G protein-coupled receptors for Drosophila PRXamide peptides, CCAP, corazonin, and AKH supports a theory of ligand-receptor coevolution. Proc. Natl. Acad. Sci. U.S.A. 99, 11423-11428. doi: 10.1073/pnas.162276199

Patel, H., Orchard, I., Veenstra, J. A., and Lange, A. B. (2014). The distribution and physiological effects of three evolutionarily and sequence-related neuropeptides in Rhodnius prolixus: adipokinetic hormone, corazonin and adipokinetic hormone/corazonin-related peptide. Gen. Comp. Endocrinol. 195, 1-8. doi: 10.1016/j.ygcen.2013.10.012

Roch, G. J., Busby, E. R., and Sherwood, N. M. (2014). GnRH receptors and peptides: skating backward. Gen. Comp. Endocrinol. 209, 118-134. doi: 10.1016/j.ygcen.2014.07.025

Semmens, D. C., Mirabeau, O., Moghul, I., Pancholi, M. R., Wurm, Y., and Elphick, M. R. (2016). Transcriptomic identification of starfish neuropeptide precursors yields new insights into neuropeptide evolution. Open Biol. 6:150224. doi: 10.1098/rsob.150224

Sha, K., Conner, W. C., Choi, D. Y., and Park, J. H. (2012). Characterization, expression, and evolutionary aspects of corazonin neuropeptide and its receptor from the house fly, Musca domestica (Diptera: Muscidae). Gene 497, 191-199. doi: 10.1016/j.gene.2012.01.052 
Tanaka, S., and Pener, M. P. (1994). A neuropeptide controlling the dark pigmentation in color polymorphism of the migratory locust, Locusta migratoria. J. Insect Physiol. 40, 997-1005. doi: 10.1016/0022-1910(94)90138-4

Tanaka, S., and Yagi, S. (1997). Evidence for the involvement of a neuropeptide in the control of body color in the desert locust, Schistocerca gregaria. Jpn. J. Appl. Entomol. Zool. 65, 447-457.

Tanaka, S. (1993). Hormonal deficiency causing albinism in Locusta migratoria. Zool. Sci. 10, 467-471.

Tanaka, S. (2000). The role of $\left[\mathrm{His}^{7}\right]$-corazonin in the control of body-color polymorphism in the migratory locust, Locusta migratoria (Orthoptera: Acrididae). J. Insect Physiol. 46, 1169-1176. doi: 10.1016/S0022-1910(99) 00228-0

Tawfik, A., Tanaka, S., De Loof, A., Schoofs, L., Baggerman, G., Waelkens, E., et al. (1999). Identification of the gregarization-associated dark-pigmentotropin in locusts through an albino mutant. Proc. Natl. Acad. Sci. U.S.A. 96, 7083-7087. doi: $10.1073 /$ pnas.96.12.7083

Veenstra, J. A. (1989). Isolation and structure of corazonin, a cardioactive peptide from the American cockroach. FEBS Lett. 250, 231-234. doi: 10.1016/00145793(89)80727-6

Veenstra, J. A. (1991). Presence of corazonin in three insect species and isolation and identification of $\left[\mathrm{His}^{7}\right]$-corazonin from Schistocerca gregaria. Peptides 12, 1285-1289. doi: 10.1016/0196-9781(91)90208-7

Veenstra, J. A. (2009). Does corazonin signal nutritional stress in insects? Insect Biochem. Mol. Biol. 39, 755-762. doi: 10.1016/j.ibmb.2009.09.008

Wigglesworth, V. B. (1934). The physiology of ecdysis in Rhodnius prolixus (Hemiptera). II. Factors controlling moulting and metamorphosis. J. Cell Sci. 77, 191-222.
Yang, J., Huang, H., Yang, H., He, X., Jiang, X., Shi, Y., et al. (2013). Specific activation of the $G$ protein-coupled receptor BNGR-A21 by the neuropeptide corazonin from the silkworm, Bombyx mori, dually couples to the $\mathrm{G}_{\mathrm{q}}$ and $\mathrm{G}_{\mathrm{s}}$ signaling cascades. J. Biol. Chem. 288, 11662-11675. doi: 10.1074/jbc.M112.441675

Zhao, Y., Bretz, C. A., Hawksworth, S. A., Hirsh, J., and Johnson, E. C. (2010). Corazonin neurons function in sexually dimorphic circuitry that shape behavioral responses to stress in Drosophila. PLoS ONE 5:e9141. doi: 10.1371/journal.pone.0009141

Žitňan, D., Kingan, T. G., Hermesman, J. L., and Adams, M. E. (1996). Identification of ecdysis-triggering hormone from an epitracheal endocrine system. Science 271, 88-91. doi: 10.1126/science.271.5245.88

Žitňan, D., Ross, L. S., Žitňanova, I., Hermesman, J. L., Gill, S. S., and Adams, M. E. (1999). Steroid induction of a peptide hormone gene leads to orchestration of a defined behavioral sequence. Neuron 23, 523-535. doi: 10.1016/S08966273(00)80805-3

Conflict of Interest Statement: The authors declare that the research was conducted in the absence of any commercial or financial relationships that could be construed as a potential conflict of interest.

Copyright (c) 2016 Hamoudi, Lange and Orchard. This is an open-access article distributed under the terms of the Creative Commons Attribution License (CC BY). The use, distribution or reproduction in other forums is permitted, provided the original author(s) or licensor are credited and that the original publication in this journal is cited, in accordance with accepted academic practice. No use, distribution or reproduction is permitted which does not comply with these terms. 